\title{
WHICH PATIENTS WITH PROSTATE CANCER ARE ACTUALLY CANDIDATES FOR HORMONE THERAPY?
}

\author{
ALESSANDRO SCIARRA, ANTONIO CARDI, GIANFILIPPO SALVATORI, GIUSEPPE \\ D'ERAMO, GIANNA MARIOTTI, FRANCO DI SILVERIO
}

Department of Urology U Bracci, University La Sapienza, Rome, Italy

\begin{abstract}
In this article, we will try to address the following aspects: which factors are responsible of the introduction of new candidates for hormone therapy in prostate cancer, who are actually candidates for hormone therapy, classifying them on the basis of the stage of the disease, and which treatment modalities can be proposed for each candidate.

Since the introduction of hormone therapy for the treatment of prostate cancer, there has been a debate about the optimal timing of hormone therapy. A modification in the timing of hormone therapy produced new candidates for hormone manipulation.

In particular, the use of hormone treatment for younger patients, longer periods and early prostate cancer, absolutely requires a whole re-evaluation of which therapy is indicated and it may produce new problems such as higher risk of over-treatment, need of a better evaluation of quality of life in younger patients and the research for better tolerated therapies. Therapies that resist for longer periods without the production of a hormone-refractory disease are also required.
\end{abstract}

Key words: prostatic neoplasms; hormone therapy; carcinoma

Int Braz J Urol. 2004; 30: 455-65

\section{INTRODUCTION}

Hormone treatment of prostate cancer is based on the demonstration that malignant prostate cells are target tissues of androgen action. Today, more than 50 years later the first evidence, endocrine manipulation remains one of the principal corner stone in the management of prostate cancer.

Unfortunately, the use of androgen deprivation in patients with prostate cancer has limitations. Most importantly, endocrine treatment can be considered palliative in nature, and relapse of the malignancy occurs if the patient survives competing with causes of death.

Different issues are still open for discussion, in particular actually new candidates for androgen manipulation are considered.
In this article, we will try to analyze which factors are responsible of the introduction of new candidates for hormone therapy, which are actually candidates for hormone therapy, classifying them on the basis of the stage of the disease, and which treatment modality can be proposed for each candidate.

\section{WHICH FACTORS DETERMINED THE INTRODUCTION OF NEW CANDIDATES FOR HORMONE THERAPY?}

In this last 20 years, different issues probably determined a new reconsideration of candidates for hormone therapy in prostate cancer.

\section{The Society}

In many developed countries, prostate cancer is the most commonly diagnosed life-threatening 
malignancy in men, and seems poised to overtake lung cancer as the major cause of cancer death (1). These aspects, added to the well known demographic shifts toward an increasingly aged society, have led epidemiologists to predict a dramatic increase in both the incidence of and death rate from prostate cancer by the year 2020, unless effective improvements in prevention, early diagnosis and treatment are forthcoming (2).

Unlikely most other forms of cancer, however, not every prostate tumor constitutes a serious threat to life of the individual affected, and consequently does not automatically warrant treatment (3).

\section{The PSA Era}

In the era of increasingly widespread prostate specific antigen (PSA) testing, the result is an early detection and early treatment of prostate cancer disease. Since the introduction of hormone therapy, there has been a debate about the optimal timing of hormone therapy. A modification in the timing of hormone therapy produced new candidates for hormone manipulation.

In particular, at the time of the Veteran's Administration Cooperative Urological Research Group (VACURG) studies, the clinicians' choice was fairly limited: they could either carry out surgical castration or give estrogens, which were associated with a high risk of complications (4). For many years, the VACURG studies formed the scientific basis for withholding endocrine therapy until symptoms appeared. An elderly man may die from an unrelated cause before he develops symptoms requiring treatment.

Other studies, in particular the Medical Research Council (MRC) (5) and the European Organization for Research and Treatment of Cancer (EORTC) 30846 (6), changed this point of view. Prostate cancer is treated at diagnosis in the hope of delaying the onset of symptoms and it may also prolong survival.

A following reason to change timing for hormone therapy has been also the introduction of the concept of biochemical progression. Several studies underlined that a PSA progression is indicative of a subsequent clinical disease progression; it can precede clinical progression by up to 4 years (7). More- over, approximately $22 \%$ of patients within 3 years of the primary local treatment will experience biochemical progression (7). The management of a rising PSA level after primary therapies with curative intent is an increasingly common problem facing clinicians. The use of hormone therapy in this setting is now common.

Very recently, moreover, the high incidence and mortality rates associated with prostate cancer have stimulated much interest in developing ways to prevent prostate cancer. Prevention medicine is a relatively new concept in urology. It may be distinguished as primary or also as secondary prevention, but both means an earlier phase to start hormone therapy and new candidates for treatment.

All these aspects may actually produce new candidates for new hormone therapy modalities, in particular younger candidates with early prostate cancer rather than advanced prostate cancer candidates and longer periods of treatment.

\section{POSSIBLE CONSEQUENCES OF HORMONE THERAPY IN EARLY PROSTATE CANCER: WHICH THERAPY FOR WHICH CANDIDATE?}

The use of hormone treatment for younger patients, longer periods and early prostate cancer, absolutely requires a whole re-evaluation of which therapy is indicated and it may produce new problems, that is, higher risk of over-treatment, need of a better evaluation of quality of life in younger patients and the research for better tolerated therapies, and therapies that resist for longer periods without the production of a hormone-refractory disease.

\section{Risk of Over-Treatment}

While no upper limit has been established over which PSA testing is not recommended, there is general agreement that men with less than a 10-year lifeexpectancy are unlikely to gain years of life for early detection and treatment is associated with an increased chance of quality of life reduction. Some elderly men in fact, die with rather than of prostate cancer, leading historically to some urologists adopting a stance of therapeutical nihilism for this malignancy $(8,9)$. 


\section{Risk of Hormone-Refractory Disease}

Our androgen deprivation therapy can select androgen independent cells or stimulate re-differentiation of transient promoter cells: the result is the development of a hormone-insensitive disease (D3). Different mechanisms can be used by prostate cancer to develop hormone-resistance, and we particularly focused on the activation of neuroendocrine prostate system.

Some experiences underlined that the longterm administration of a continuous complete androgen deprivation (CAD) therapy produces a progressive increase in chromogranin $\mathrm{A}(\mathrm{CgA})$ (10). It should be possible that continuous $\mathrm{CAD}$ may determine a hyperactivation of neuroendocrine (NE) system in the prostate gland. This may be one of the mechanisms used by the prostate adenocarcinoma to progress during hormone therapy in an androgen-insensitive disease.

We published data demonstrating a significant increase in tissue mRNA and serum expression of $\mathrm{CgA}$ after 3 or more months of CAD therapy for prostate cancer (11). If longer periods of hormone therapy will be used, new treatment modalities that resist without the production of a hormone-insensitive disease are needed. Also, from this point of view, the introduction and comparison of an intermittent versus continuous administration of the therapy or the use of antiandrogen monotherapy versus castration, all either as primary, adjuvant or neoadjuvant treatments, can be better analyzed.

We published results from a prospective randomized trial, showing that the intermittent administration when compared with the continuous administration of CAD and non-steroidal antiandrogen monotherapy compared to castration therapy, may prevent or reduce the risk of $\mathrm{CgA}$ increase and $\mathrm{NE}$ hyperactivation during treatments $(12,13)$ (Figure-1).

\section{Quality of Life Considerations}

As the result of the use of hormone therapy in the management of patients with early prostate cancer, there is a strong likelihood of patients receiving this therapy for a longer duration (14). Therefore,

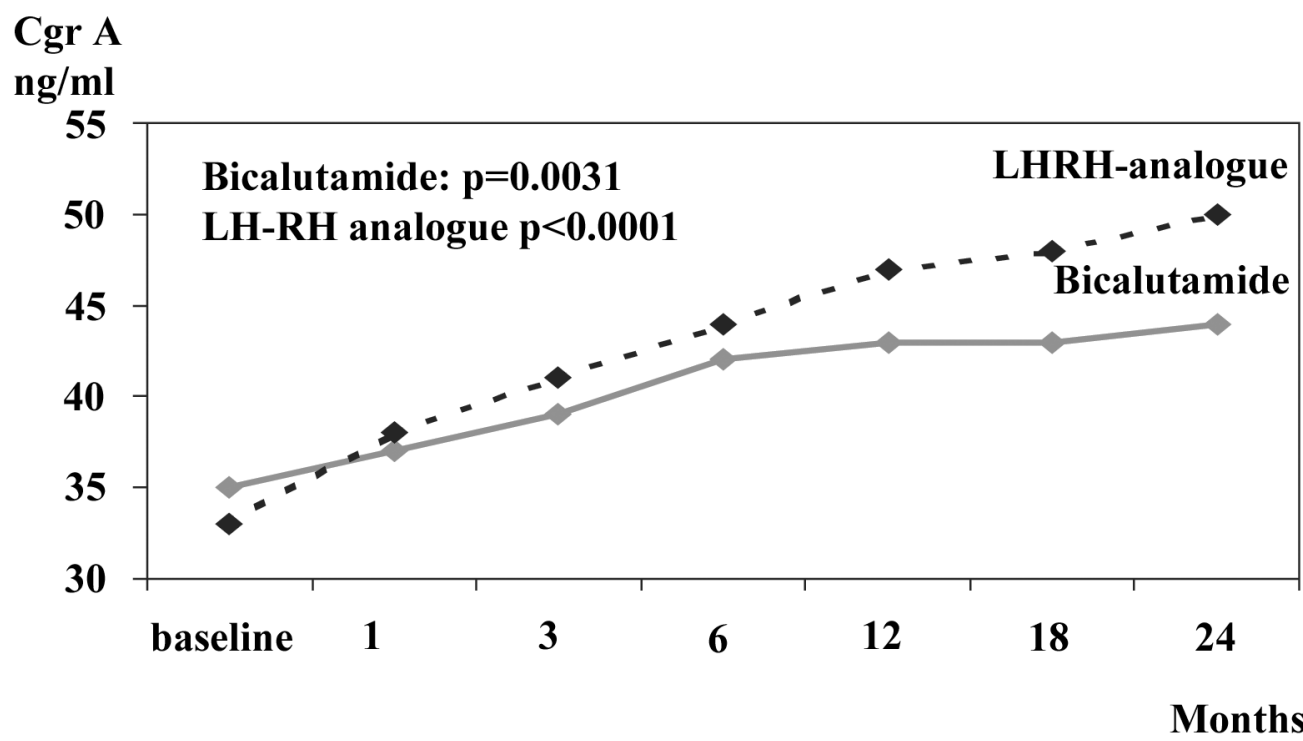

Figure 1 - Effect of castration therapy versus antiandrogen monotherapy on chromogranin $A(C g A)$ expression in locally advanced prostate cancer (ref. 13). 
a major consideration in the choice of hormone therapy is the physiological and psychological impact it has on patients. For this reason in the last International Consultation on Prostate Cancer, our Committee underlined some questions (15):

1) Under the consideration of the new candidates for hormone therapy, is it not better to initiate endocrine treatment in a less aggressive fashion and step it up later or when progression occurs?

2) If quality of life can really be improved for a prolonged period of time and the therapies were less effective in terms of disease specific and overall survival, would patients be ready to exchange quality of life for lifetime?

On the basis of all these considerations, we will try to analyze the actual indication for hormone therapy for each stage of the disease.

\section{CANDIDATES FOR HORMONE THERAPY: LOCALIZED PROSTATE CANCER}

In the localized stage of prostate cancer the first question is: Is there a role for a deferred hormone treatment, that means no initial treatment with close surveillance followed by active treatment when and if the tumor progresses with symptoms?

In this stage, the rational for deferring treatment is the often-protracted course of localized prostate cancer and the fact that many patients with such tumors die with the disease and not of it (16). The result of he Surveillance Epidemiology and End Re- sults Program from USA (17), analyzing 19,898 patients managed conservatively, was that the overall disease-specific survival rate 10 -year after diagnosis was $90 \%$ for those with well or moderately differentiated tumors, but at 10-years the metastasis free survival was lower for cases with moderately differentiated compared to those with well differentiated tumors (17). The conclusion of the International Consultation on Prostate Cancer (16) is that deferred treatment may be an option for patient with clinically localized low-grade prostate cancer with a life expectancy of 10 to 15 years or less.

However, indications for hormone therapy in localized prostate cancer are restricted as described in (Table-1). A possible indication for hormone therapy in this stage of the disease is as neoadjuvant treatment prior to radiation or surgical therapy. The rationale for neoadjuvant hormonal therapy is the induction of early regression of the primary tumor bulk and a coincided treatment of micrometastatic disease.

However, the use of neoadjuvant hormone therapy prior to radical prostatectomy is highly controversial. As concluded by the International Consultation on Prostate Cancer (16), the only positive result suggested for a hormone therapy neoadjuvant to radical prostatectomy, seems to be a reduction of positive surgical margins in clinical T2 tumors.

More positive results are described for the use of neoadjuvant therapy prior to radiotherapy, but longer follow-up and more data in terms of survival are needed (18).

Table 1 - Role of hormone therapy in localized prostate cancer. Recommendation of the International Consultation on Prostate Cancer (ref. 7).

Role of Hormonal Therapy in Localized Prostate Cancer

\begin{tabular}{ll}
\hline Stage T1a or T1b & No reported date available \\
Stage T1c-T2 & - Patient's refusal of active treatment \\
& - Older patient's with less than 5 years life-expectancy who refuse watchful waiting \\
& - Older men who are unable to undergo radical therapy \\
& - Comorbid conditions which preclude definitive therapy \\
& - Failure of previous definitive local therapy \\
\hline
\end{tabular}


The recommendations of the International Consultation on prostate cancer regarding as the neoadjuvant hormone therapy have been:

- it seems to yield improved results in terms of local control in cases considered for radiotherapy

- no demonstrated benefits prior to radical prostatectomy have been demonstrated; longer period of neoadjuvant hormone therapy may be analyzed (19).

\section{CANDIDATES FOR HORMONE THERAPY: LOCALLY ADVANCED PROSTATE CANCER}

In this last years the use of different formulations of hormone therapy in locally advanced prostate cancer has been strongly analyzed.

The recommendations of the international consultation (7) are referred to locally advanced disease as T3-T4 N0-M0 or T1-T4 N1-M0. In unscreened populations, over $30 \%$ of all patients present with locally advanced disease (7). The current treatment recommended for this stage lists radical prostatectomy, external beam radiotherapy and hormone therapy (Figures-2 and 3). However, the information of several phase II studies suggests that in patients treated with radical prostatectomy or radiotherapy, adjuvant hormone therapy improves disease-free and overall survival $(7,20,21)$.

Fewer data are in favor of hormone therapy alone and, in this case, a deferred treatment has been considered only for Gleason score $<7$, older age, and asymptomatic cases $(7,22,23)$.

Approximately $30-50 \%$ of patients with clinical T3-T4 tumors are found to have pathologically involved pelvic lymph nodes $(24,25)$. Several phase II trials suggested an improved outcomes in patients treated with radiotherapy and hormone therapy or radical prostatectomy and hormone therapy, but no

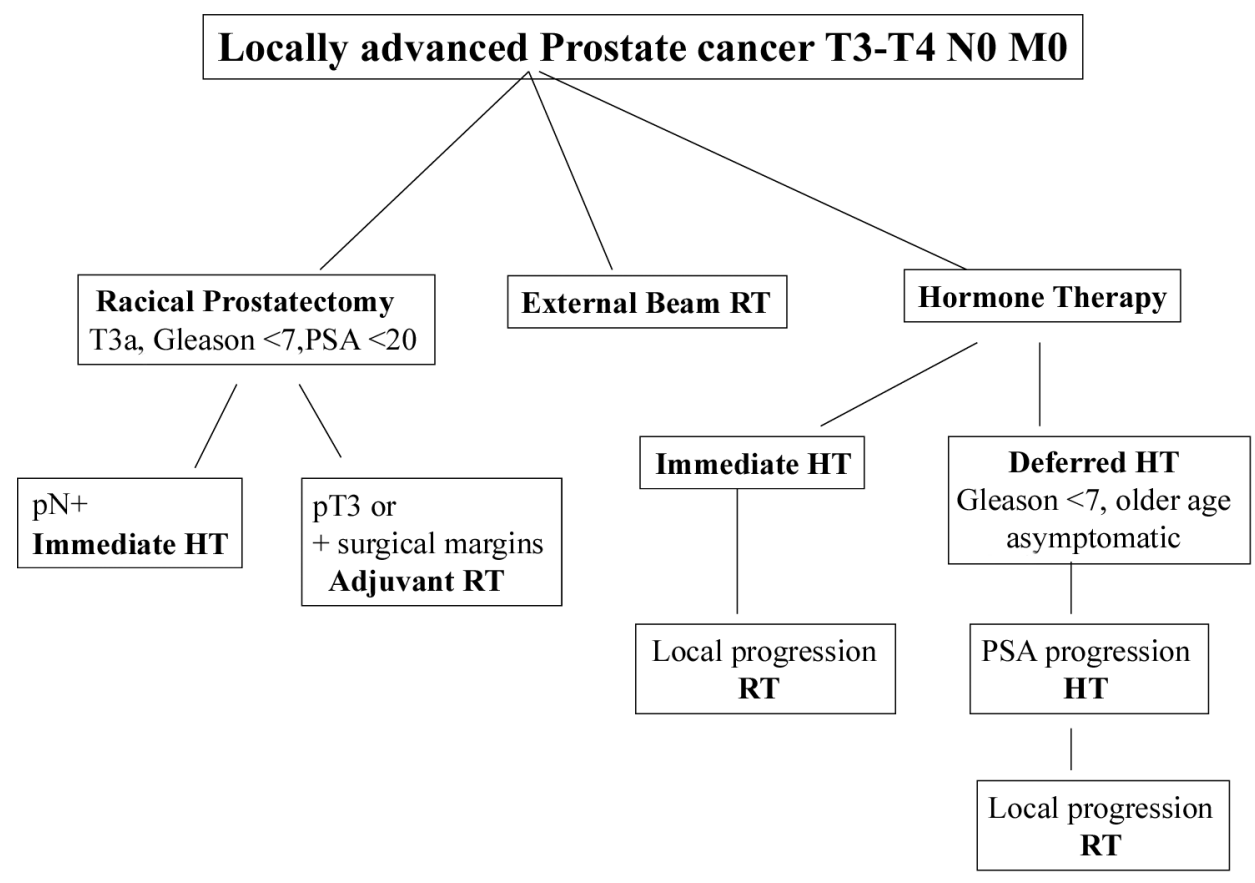

Figure 2 - Treatment options for T3-T4 NO MO prostate cancer candidates. Recommendation of the International Consultation on Prostate Cancer (ref. 7). HT = hormone therapy; $R=$ radiotherapy. 


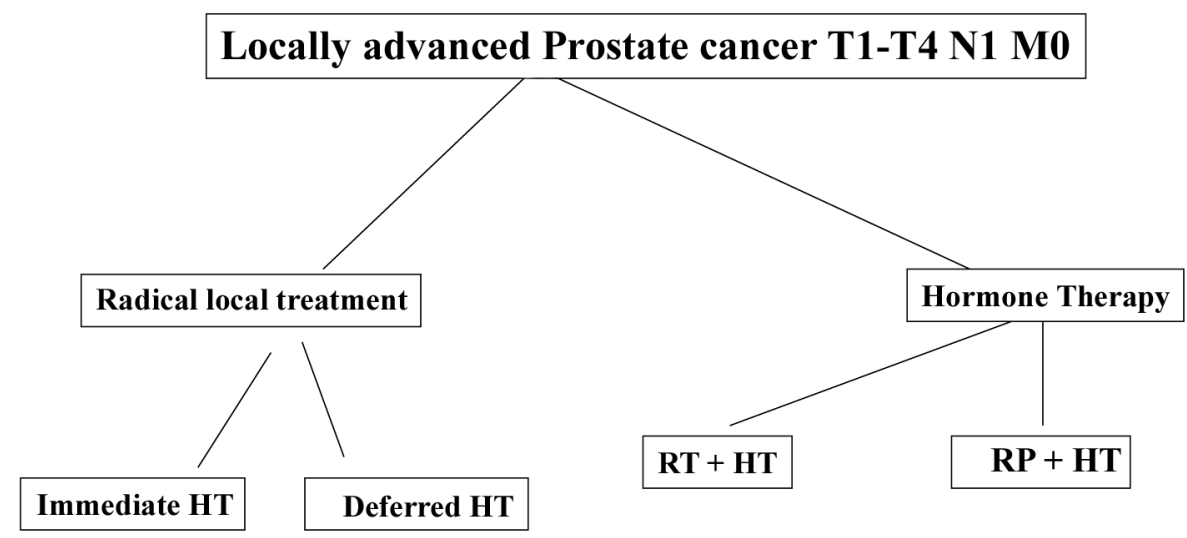

Figure 3 - Treatment options for T1-T4 N+ M0 prostate cancer candidates. Recommendation of the International Consultation on Prostate Cancer (ref. 7). HT = hormone therapy; $R T$ = radiotherapy.

good evidence from phase III randomized trials is available to support a routine use of combined modalities in patients with lymph nodes involvement $(7,26,27)$. Messing et al. (28) reported results of a randomized phase III trial of immediate versus deferred hormone therapy in $\mathrm{N}+$ cases treated with radical prostatectomy. At 7.2 years of follow-up, the overall risk of death was 13\% in the group who received immediate hormone therapy versus $34 \%$ in those in the deferred group. However, in the absence of perfect evidence, clinicians who make treatment recommendations to patients with locally advanced prostate cancer, should be aware of the range of treatment options available (7).

From the point of view of quality of life results, in recent years, discussion on alternative hormone therapy modalities for locally advanced disease has been developed.

Antiandrogens may represent a treatment modality which given as monotherapy, is associated with few adverse effects. In particular the comparative analysis of the 480 non-metastatic patients with locally advanced T3-T4 M0 disease in the 2 randomized studies of bicalutamide $150 \mathrm{mg}$ as monotherapy and castration, has been performed after a median follow-up of 6.3 years and a $56 \%$ deaths $(30,31)$. Bicalutamide monotherapy was statistically equivalent to castration in terms of overall survival and it was possible to estimate median survival, which was 63.5 months in bicalutamide and 69.9 months in the castration group. No significant difference in terms of time to progression between the 2 groups was found. On the other hand, most of the parameters related to quality of life showed a positive treatment effect in favor of bicalutamide, with sexual interest and physical capacity showing statistically significant advantages compared to castration (31).

\section{Adjuvant Hormone Therapy in Locally Advanced Disease}

Most of locally advanced candidates for hormone therapy will receive the therapy as an adjuvant treatment. There is now considerable interest in the use of adjuvant hormone therapy after primary treatment. Adjuvant therapy may have a role although not all patients can be expected to benefit.

The bicalutamide early prostate cancer program (EPC) is being undertaken to investigate the efficacy of bicalutamide as an adjuvant therapy of primary curative intent or as immediate hormone therapy in men with non-metastatic prostate cancer. A total of 8,113 men have been considered. Patients were assigned in a 1:1 ratio to receive either bicalutamide 150 $\mathrm{mg}$ or placebo. See et al. reported results on 3,603 cases as part of the EPC program (32), the median follow-up was 2.6 years. A significant reduction of $43 \%$ was found in the risk of objective progression for bicalutamide compared with placebo. This benefit was consistent regardless of whether bicalutamide was given as adjuvant therapy or after watchful waiting. The survival 
data were immature. It is important to analyze the role of adjuvant hormone therapy in T3 tumors for each primary therapy used.

Bolla et al. (21) reported improved overall survival with adjuvant hormone therapy added to radiotherapy versus radiotherapy alone, on T1-T4 diseases (goserelin for 3 years) $(\mathrm{HR}=0.50 ; 95 \% \mathrm{CI}=$ $0.33-0.76 ; p=0.001)$. However other studies, for example Radiation Therapy Oncology Group (RTOG) 92-03 on T3 tumors showed that adjuvant therapy (goserelin during the last week of radiotherapy until progression) significantly improved clinical local control (85\% versus $71 \%$ in radiotherapy alone) and disease free survival ( $60 \%$ versus $44 \%$ ), but not overall survival.

If we stratify the results of EPC program in patients who underwent radiotherapy as primary therapy, the highest advantage of adjuvant therapy in terms of reduction of progression, was obtained in locally advanced, $\mathrm{N}+$ and moderately to poorly differentiated tumors (32).

Similarly, stratifying cases of the EPC program who underwent radical prostatectomy as primary treatment, the advantage of adjuvant therapy on placebo was significant in locally advanced, $\mathrm{N}+$, Gleason score $>7$ tumors (32).

Therefore, the conclusion of our Committee in the International Consultation on Prostate Cancer (15) was that, as nonsteroidal antiandrogen are associated with potential quality of life benefits when compared to castration, in early prostate cancer candidates to hormone therapy, it may be preferable to use antiandrogen as first line therapy and then step up therapy with the use of more invasive forms of hormone deprivation. However, at now, the answer to the question "must we treat all cases submitted to radiotherapy or radical prostatectomy with adjuvant hormone therapy", is negative.

\section{CANDIDATES FOR HORMONE THERAPY: ADVANCED PROSTATE CANCER}

Patients with advanced prostate cancer represent the traditional candidates for hormone therapy. Despite recent advances in the early detection of pros- tate cancer, $10 \%$ to $50 \%$ of clinically localized prostate cancer will progress (34). Our concept of incurable or advanced prostate cancer has changed considerably in recent years. It was proposed that the definition of advanced prostate cancer must include not only soft tissue or bone metastases $(\mathrm{M}+)$, but also stage D1 as a rising PSA after failed local therapy. Men presumed to have localized prostate cancer, now undergo neoadjuvant and adjuvant hormone therapies. Their response to subsequent hormone therapy may not be the same as a male newly diagnosed with metastatic disease who has not received prior hormone therapy (7). Thus, more men who have had prior hormone manipulations are drifting to advanced disease. Prior studies have confirmed that an initial response may be obtained in $60 \%$ to $80 \%$ of untreated metastatic prostate cancer. However, in men who have previously received treatment, this may not be the case (7).

Also in patients with advanced metastatic disease the quality of life issue is important in the decision of treatment. Hormone therapy is the principal treatment strategy for metastatic prostate cancer, however, discussion on timing and on the type of androgen deprivation is actual.

In men with symptoms or with complications, immediate treatment is mandatory. Even without evidence of improved survival, Medical Research Council Prostate Cancer Working Party Investigator Group (MRC) study in M1 (5), additional arguments favor immediate treatment in most patients with asymptomatic metastases. Delay before an indication for treatment occurs, will be brief; $50 \%$ of M1 patients were treated within 9 months of entry in MRC study (5). The excess of complications such as spinal cord compression and pathological fractures in deferred treatment patients was mainly seen in those with M1 disease at presentation. This increased risk may not be reduced when the deferred treatment is commenced. Deferring treatment also increases the early risk of local progression sufficient to require transurethral resection of the prostate (36).

Therefore the recommendation of the International Consultation on Prostate Cancer (30) are that patients with metastases should be advised to commence hormone therapy immediately at diagnosis. If 
treatment is deferred, this should be in well motivated patients with low risk disease, such as small number of low density metastatic hot spots on scintigraphy and low PSA (30).

The discussion is also pointed on which therapy for these candidates. Two different modalities of hormone therapy has been analyzed so to improve quality of life and reduce toxicity from therapy in metastatic prostate cancer: non-steroidal antiandrogen monotherapy and intermittent androgen deprivation (IAD).

In protocol 306 and 307 comparing bicalutamide monotherapy with castration, data on metastatic cases were considered mature as $43 \%$ of M1 cases had died (31). Bicalutamide $150 \mathrm{mg}$ monotherapy resulted inferior to castration with respect to time to death. However, the difference in median survival between the 2 treatment groups was only 6 weeks. Again, most of the parameters on quality of life, including sexual function, showed a positive treatment effect in favor of bicalutamide (31).

IAD therapy has been proposed as primary therapy in M1 cases (37), but also as adjuvant to radi- cal prostatectomy (38) or radiotherapy (39) in locally advanced cases.Few randomized trials compared IAD to continuous CAD in metastatic disease. The recommendation of the International Consultation on Prostate Cancer (30) is that IAD must be yet considered an investigational regimen.

\section{NEW CANDIDATES FOR HORMONE THERAPY}

Patients with androgen deprivation therapy refractory prostate cancer (D3 stage) are men with poor prognosis, limited survival and limited chances of response to conventional therapies. In these cases also salvage chemotherapy cannot extent survival of more than 10 months (40).

It has been hypothesize that some factors may act as "survival factors". Survival factors may interfere in the apoptotic processes, conferring a sort of immortalization to the neoplastic cell. In particular insuline-like growth factor (IGF) may act as survival factor for prostate adenocarcinoma cells. NE differentiation and activity may be another factor

\section{Rationale of our combination therapy}

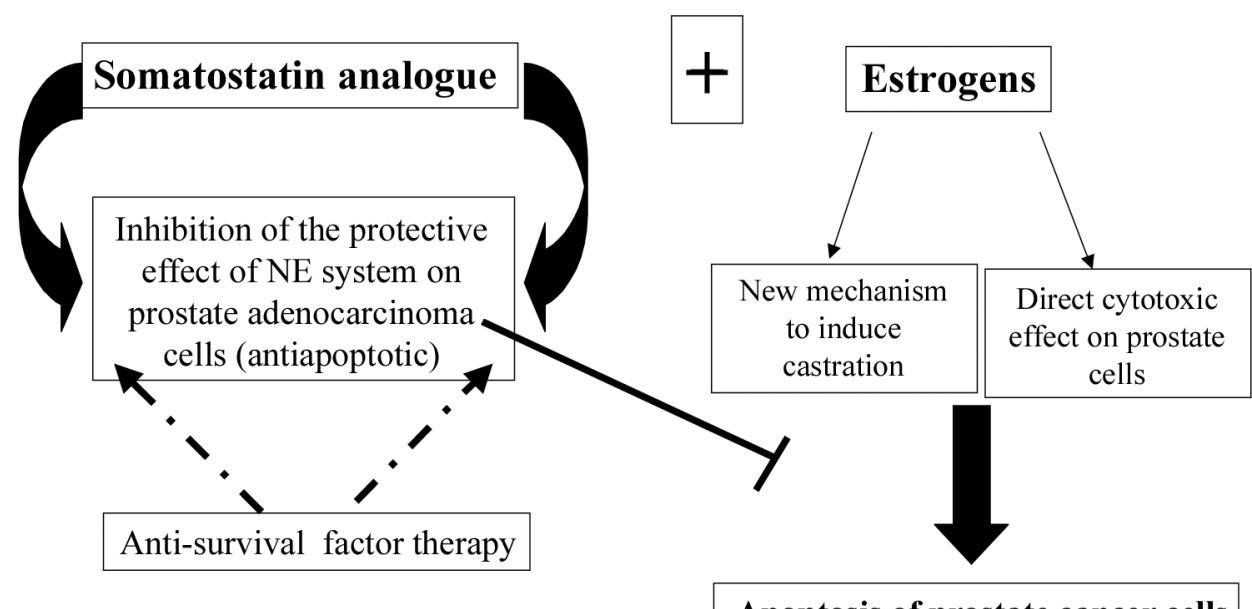

Apoptosis of prostate cancer cells

Figure 4 - Antisurvival combination therapy for advanced prostate cancer. 
used by prostate adenocarcinoma to progress during hormone therapy in an androgen insensitive disease. NE cells can produce and release several peptides that can protect the prostate adenocarcinoma cell from apoptosis. We and other authors $(40,41)$ proposed the use of "antisurvival factor therapies" for the treatment of D3 prostate cancer. Koutsilieris et al. (40) proposed the combination therapy with somatostatin analogue and dexametasone so to prevent the effect of IGF on D3 prostate cancer and to restore a sensibility to androgen deprivation therapy. We proposed the combination therapy of somatostatin analogue (lanreotide acetate) and ethinylestradiol in D3 tumors (41) (Figure-4). The rationale of our combination therapy is: somatostatin analogue may act reducing NE activity and NE peptides release from NE prostate cells. In this way somatostatin analogue may reduce the activity of the survival factors produced by the NE component and therefore may restore a normal apoptotic process. We used estrogen as second line therapy after progression from CAD therapy, and so to add a direct cytotoxic effect from estrogens on prostate tumor cell (41). It is important to underline that antisurvival therapies cannot be used as monoterapy but only in combination therapy with agents that can produce a direct cytotoxic effect on prostate cancer cells. Somatostatin analogue, reducing IGF activity or NE activity on the prostate gland, may produce a new response to androgen deprivation therapy.

Therefore, it is possible that new candidates for hormone therapy will be those that today are considered androgen refractory D3 tumors but that instead represent cases with only a reduced androgen sensitivity.

\section{REFERENCES}

1. Parker SL, Tong T, Bolden S, Wingo PA: Cancer Statistic 1997. CA Cancer J Clin. 1997; 47: 5-27. Erratum in: CA Cancer J Clin. 1997; 47: 68.

2. Carter HB, Coffey DS: The prostate: an increasing medical problem. Prostate. 1990; 16: 39-48.

3. Kirby RS, Christmas TJ, Brawer MK: Prostate Cancer, 2nd International Consultation on Prostate Cancer. Paris, Mosby. 1999; pp. 21-56.
4. Byar DP: Proceedings: The Veterans Administration Cooperative Urological Research Group's studies of cancer of the prostate. Cancer. 1973; 32: 112630.

5. The Medical Research Council Prostate Cancer Working Party Investigator Group. Immediate versus deferred treatment for advanced prostatic cancer: initial results of the Medical Research Council Trial. Br J Urol. 1997; 79: 235-46.

6. Denis LJ, Keuppens F, Smith PH, Whelan P, de Moura JL, Newling D, et al.: Maximal androgen blockade: final analysis of EORTC phase III trial 30853. EORTC Genito-Urinary Tract Cancer Cooperative Group and the EORTC Data Center. Eur Urol. 1998; 33: $144-51$.

7. Kirby RS, Christmas TJ, Brawer MK: Prostate Cancer, 2nd International Consultation on Prostate Cancer. Paris, Mosby. 1999, pp. 327-350.

8. Wilt TJ, Brawer MK: The prostate cancer intervention versus observation trial (PIVOT). Cancer. 1994; 75: 1963-68.

9. Barre M, Nerstrom B, Overgaard J: The natural history of prostate carcinoma based on a Danish population treated with no attempt to cure. Cancer. 1997; 80: 917-28.

10. Monti S, Sciarra A, Falasco P: Serum concentrations and prostatic gene expression of Chromogranin A and PSA in patients affected by prostate cancer and benign prostatic hyperplasia. J Endocrin Invest. 2000; 23 (Suppl. 8): 53A.

11. Sciarra A, Mariotti G, Gentile V, Pastore A, Voria G, Di Silverio F, et al.: Neuroendocrine differentiation in human prostate tissue: is it detectable and treatable? BJU Int. 2003; 91: 438-45.

12. Sciarra A, Monti S, Gentile V, Mariotti G, Cardi A, Voria $\mathrm{G}$, et al.: Variation in chromogranin A serum levels during intermittent versus continuous androgen deprivation therapy for prostate adenocarcinoma. Prostate. 2003; 55: 168-79.

13. Sciarra A, Gentile V, Pastore A, Voria G, Mariotti G, Di Silverio F, et al.: Effect of nonsteroidal antiandrogen monotherapy versus castration therapy on neuroendocrine differentiation in prostate carcinoma. Eur Urol. 2003; 49 (Suppl. 2): 742A.

14. Schroder FH: Antiandrogen as monotherapy for prostate cancer. Eur Urol. 1998; 34 (Suppl. 3): 12-7.

15. Debruyne FMJ, Akaza H, Klotz L: Innovative Approaches in Medical Management of Prostate Cancer: Hormones, 5th International Consultation on Prostate Cancer. Paris, J.R. Spermon. 2003. 
16. Kirby RS, Christmas TJ, Brawer MK: Prostate Cancer, 2nd International Consultation Prostate Cancer. Paris, Mosby. 1999; pp. 263-326.

17. Lu Yao GL, Yao SL: Population-based study of long term survival in patients with clinically localized prostate cancer. Lancet. 1997; 349: 906-10.

18. Roach M: Neoadiuvant therapy prior to radiotherapy for clinically localized prostate cancer. Eur Urol. 1997; 32 (Suppl. 3): 48-54.

19. Gleave ME: Randomized, comparative study of 3 vs 8 months of neoadjuvant hormonal therapy prior to radical prostatectomy. Biochemical and pathological effects. J Urol. 1999; 161 (Suppl.): 592A.

20. Lerner SE, Blute ML, Zincke H: Extended experience with radical prostatectomy for clinical stage T3 prostate cancer: outcomes and contemporary morbidity. J Urol. 1995; 154: 1447-52.

21. Bolla M, Gonzalez D, Warde P, Dubois JB, Mirimanoff RO, Storme G, et al.: Improve survival in patient with locally advanced prostate cancer treated with radiotherapy and goserelin. N Engl J Med. 1997; 337: 295-300.

22. Gomez JL, Cusan L, Diamond P, Candas B, Labrie F: Long term treatment of clinical stage $\mathrm{C} / \mathrm{T} 3$ prostate cancer with flutamide and castration: 6 year median follow-up. Br J Urol. 1997; 80 (Suppl. 2): 275.

23. Rana A, Crisholm GD, Khan M, Rashwan HM, Elton RA: Conservative management with symptomatic treatment and delayed hormonal manipulation is justified in men with locally advanced carcinoma of the prostate. Br J Urol. 1994; 74: 637-41.

24. van den Ouden D, Davidson PJ, Hop W, Schroder FH: Radical prostatectomy as a monotherapy for locally advanced (stage T3) prostate cancer. J Urol. 1994; 151: 646-51.

25. Zincke H, Utz DC, Taylor WF: Bilateral pelvic lymphadenectomy and radical prostatectomy for clinical stage $\mathrm{C}$ prostatic cancer: role of adjuvant treatment for residual cancer and disease progression. J Urol. 1986; 135: 1199-205.

26. Seay TM, Blute ML, Zincke H: Long term outcome in patient with pTx $\mathrm{N}+$ adenocarcinoma of prostate treated with radical prostatectomy and early androgen ablation. J Urol. 1998; 159: 357-64.

27. Ghavamian R, Bergstralh EJ, Blute ML, Slezak J, Zincke H: Radical retropubic prostatectomy plus orchiectomy versus orchiectomy alone for pTx N+ prostate cancer: a matched comparison. J Urol. 1999; 162: 1223-7.

28. Messing E, Manola J, Wilding G, Sarosdy M; Crawford D; Trump D: Immediate hormonal therapy vs observa- tion for node positive prostate cancer following radical prostatectomy and pelvic limphadenectomy: a randomized phase III Eastern Cooperative Oncology Group / Inter Group Trial. J Urol. 1999; 161 (Suppl.): 175.

29. Schroder FH: Endocrine treatment of prostate cancer - recent developments and the future. Part 1: maximal androgen blockade, early vs delayed endocrine treatment and side-effects. BJU Int. 1999; 83: 161-70.

30. Kirby RS, Christmas TJ, Brawer MK: Prostate Cancer, 2nd International Consultation on Prostate Cancer. Paris, Mosby. 1999; pp. 351-395.

31. Tyrrell CJ, Kaisary AV, Iversen P, Anderson JB, Baert $\mathrm{L}$, Tammela $\mathrm{T}$, et al.: A randomised comparison of 'Casodex' (bicalutamide) $150 \mathrm{mg}$ monotherapy versus castration in the treatment of metastatic and locally advanced prostate cancer. Eur Urol. 1998; 33: 447-56.

32. See WA, McLeod DG, Wirth MP, Iversen P, Klimberg I, Gleason D, et al.: Bicalutamide ("Casodex") 150mg as immediate or adjuvant therapy in 8113 men with localized or locally advanced CaP. J Urol. 2001; 165 (Suppl. 5): 1594A.

33. Swedish Breast Cancer Cooperative Group: Randomized trial of two versus five years of adjuvant tamoxifene for postmenopausal early stage breast cancer. J Natl Cancer Inst. 1996; 88: 1543-9.

34. Parkin DM, Pisani P, Ferlay J: Global cancer statistics. CA Cancer J Clin. 1999; 49: 33-64.

35. Varricchio CG, McCabe MS, Trimble E, Korn EL: Quality of Life in Clinical Cancer Trials. Introduction. J Natl Cancer Inst Monogr. 1996; 20: vii-viii.

36. Kirk D,On behalf of the Medical Reserch Council Prostate Working Party Investigator Group Medical Research Council: Immediate versus deferred treatment study: how important is local progression in advanced prostate cancer? Br J Urol. 1998; 81 (Suppl. 4): 30A.

37. Oliver RTD, Williams G, Paris AMI: Intermittent androgen deprivation after PSA-complete response as a strategy to reduce induction of hormone-resistant prostate cancer. Urology. 1997; 49: 79-82

38. Sciarra A, Di Chiro C, Di Silverio F: Intermittent androgen deprivation (IAD) in patients with biochemical failure after radical retropubic prostatectomy (RRP) for clinically localized prostate cancer. World J Urol. 2000; 18: 392-400.

39. Grossfeld GD, Small EJ, Carrell PR: Intermittent androgen deprivation for clinically localized prostate cancer: initial experience. Urology. 1998; 51: 13744. 
40. Koutsilieris M, Mitsiades C, Dimopoulos T, Ioannidis A, Ntounis A, Lambou T: A combination therapy of dexamethasone and somatostatin analog reintroduces objective clinical responses to LHRH analog in androgen ablation-refractory prostate cancer patients. J Clin Endocrinol Metab. 2001; 86: 5729-36.
41. Sciarra A, Gentile V, Voria G, Pastore A, Mariotti G, Di Silverio F, et al.: A combination therapy with ethinylestradiol and lanreotide reintroduce responsiveness in androgen ablation therapy in refractory prostate cancer. Eur Urol. 2003; 1: (Suppl 2), 739A.

Received: September 20, 2004 Accepted: September 30, 2004

Correspondence address:

Dr. Alessandro Sciarra

V. Nomentana 233

00161 Rome, Italy

Fax: + 3906 446-1959

E-mail: sciarrajr@hotmail.com 